\title{
New indication for a dichotomy in the interior structure of Uranus and Neptune from the application of modified shape and rotation data Accepted to Planet. Space Sci., June 2012
}

\author{
N. Nettelmann ${ }^{\mathrm{a}}$, R. Helled ${ }^{\mathrm{b}}$, J. J. Fortney ${ }^{\mathrm{c}}$, R. Redmer ${ }^{\mathrm{a}}$ \\ ${ }^{a}$ Institute for Physics, University of Rostock, 18051 Rostock, Germany \\ ${ }^{b}$ Department of Geophysics and Planetary Science, Tel-Aviv University Ramt-Aviv, Tel-Aviv 61390, Israel \\ ${ }^{c}$ Department of Astronomy and Astrophysics, University of California, Santa Cruz, CA 95064, USA
}

\begin{abstract}
Since the Voyager fly-bys of Uranus and Neptune, improved gravity field data have been derived from long-term observations of the planets' satellite motions, and modified shape and solid-body rotation periods were suggested. A faster rotation period $(-40 \mathrm{~min})$ for Uranus and a slower rotation period $(+1 \mathrm{~h} 20)$ of Neptune compared to the Voyager data were found to minimize the dynamical heights and wind speeds. We apply the improved gravity data, the modified shape and rotation data, and the physical LM-R equation of state to compute adiabatic three-layer structure models, where rocks are confined to the core, and homogeneous thermal evolution models of Uranus and Neptune. We present the full range of structure models for both the Voyager and the modified shape and rotation data. In contrast to previous studies based solely on the Voyager data or on empirical EOS, we find that Uranus and Neptune may differ to an observationally significant level in their atmospheric heavy element mass fraction $Z_{1}$ and nondimensional moment of inertia, $\lambda$. For Uranus, we find $Z_{1} \leq 8 \%$ and $\lambda=0.2224(1)$, while for Neptune $Z_{1} \leq 65 \%$ and $\lambda=0.2555(2)$ when applying the modified shape and rotation data, while for the unmodified data we compute $Z_{1} \leq 17 \%$ and $\lambda=0.230(1)$ for Uranus and $Z_{1} \leq 54 \%$ and $\lambda=0.2410$ (8) for Neptune. In each of these cases, solar metallicity models $\left(Z_{1}=0.015\right)$ are still possible. The cooling times obtained for each planet are similar to recent calculations with the Voyager rotation periods: Neptune's luminosity can be explained by assuming an adiabatic interior while Uranus cools far too slowly. More accurate determinations of these planets' gravity fields, shapes, rotation periods, atmospheric heavy element abundances, and intrinsic luminosities are essential for improving our understanding of the internal structure and evolution of icy planets.
\end{abstract}

Keywords: Uranus; Neptune; Planetary Interiors; Planetary Evolution

\section{Introduction}

The outer planets Uranus and Neptune are mysterious in many ways. While their names 'ice giants' suggest a composition of predominantly volatiles in ice phases such as water, methane, and ammonia ice, interior models instead predict a warm interior devoid of solid ices (Chau et al., 2011; Redmer et al., 2011). Structure models of Uranus and Neptune are generally in agreement in predicting a small rock core, a deep interior of more than $70 \%$ heavy elements, and a significantly less enriched outer envelope with a transition at about $70 \%$ of the radius for both planets (Hubbard et al., 1995; Fortney and Nettelmann, 2010; Helled et al., 2011). However, they also agree in failing to explain Uranus' measured intrinsic luminosity (Podolak et al., 1991). Uranus' low luminosity is a riddle; even more so as the corresponding models for Neptune nowadays can reproduce its measured luminosity (Fortney et al., 2011) indicating that the ice giants are not so similar to each other as previously thought (Podolak et al., 1995).

Contrary to the efforts that have been made to mea- sure the atmospheric composition, the Voyager 2 radio occultation data and ground-based observational microwave data have actually raised more questions than they were intended to solve. In both planetary atmospheres for instance, D/H appears enriched over the protosolar values (de Bergh et al., 1986, 1990) suggesting particle transport between the atmosphere and an ice-rich deep interior (Hubbard et al., 1995), while the inferred helium abundance is consistent with the protosolar value and thus would suggest the opposite (i.e., inefficient particle transport) if hydrogen and helium occur in the deep interior where they might undergo phase separation (Hubbard et al., 1995; Podolak et al., 1995). Moreover, for the interior of Uranus we cannot rule out a primary composition of silicates dissolved into hydrogen-helium envelopes as that might sufficiently accelerate Uranus' cooling time (Hubbard and MacFarlane, 1980). We just do not know how similar Uranus and Neptune really are in terms of composition and structure (Podolak et al., 2000).

In addition, Uranus and Neptune have complex multipolar magnetic fields, and appear to have stronger atmo- 
spheric winds than Jupiter and Saturn, if the Voyager rotation periods are applied. Besides the natural desire to understand the giant planets in our solar system, learning about the 'ice giants' is important for the classification of extrasolar planets with similar masses and sizes, as many ones are observed (Borucki et al., 2011), although not yet on similar orbital distances (Kane, 2011).

Information on Uranus and Neptune interiors are typically derived from theoretical models which are designed to fit the observed physical data of the planets, such as their gravitational fields, masses, internal rotation, and radii. The physical data available for Uranus and Neptune are rather limited. In particular, the low-order gravitational harmonics $J_{2}$ and $J_{4}$ of Neptune have significant error bars, Neptune's equatorial 1-bar radius is actually not measured, and the shapes (flattening) of both Uranus and Neptune are not well known. Voyager 2 provided only one occultation radius at the 1 bar pressure level for each planet (see Helled et al., 2010, for details). Although stellar occultations do provide information on the planetary oblateness, the shape is inferred for upper atmosphere (microbar pressure levels) and it is unclear whether this shape is consistent with the shape at the 1 bar pressure level. Helled et al. (2010) have shown that minimization of wind velocities or dynamic heights of the 1 bar isosurfaces (Anderson and Schubert, 2007; Helled et al., 2009) constrained by Voyager 2 occultation radii and gravitational coefficients of the planets, leads to modified solid-body rotation periods of $16 \mathrm{~h} 34 \mathrm{~m}$ for Uranus and $17 \mathrm{~h} 27 \mathrm{~m}$ for Neptune, which is $40 \mathrm{~min}$ shorter than the Voyager rotation period for Uranus, and 1h20 longer for Neptune. Helled et al. (2010) also state that both planets may have different rotation periods than the ones derived by the minimization method, and in addition, could be rotating differentially on cylinders. Non-solid body rotation can lead to a change in the calculated gravitational moments (Hubbard et al., 1991). Our results are valid as long as differential rotation in Uranus and Neptune is "shallow", i.e., the region of differential rotation consists of a negligible mass, which is a preferred solution for Uranus and Neptune (Y. Kaspi, priv. comm.). However, the suggested solid-body rotation periods better match the measured radii of the planets and result in more moderate atmosphere dynamics with wind velocities of $\sim 150 \mathrm{~m} \mathrm{~s}^{-1}$ for both planets. Based on these suggested rotation rates and occultation radii, modified shapes of the planets were derived. Since the shapes of Uranus and Neptune are not well constrained, and in addition, their rotation profiles must not be that of a solidbody, the modified solid-body rotation periods and shapes can be used to demonstrate the sensitivity of the interior models to the uncertainties in these physical properties.

To obtain pressure-density relations for the interior of Uranus and Neptune, three different methods have been invoked. Podolak et al. (1995) apply physical equations of state of hydrogen, helium, the ices $\mathrm{H}_{2} \mathrm{O}, \mathrm{H}_{2} \mathrm{~S}, \mathrm{CH}_{4}$, and $\mathrm{NH}_{3}$, and rocks, assuming sharp transitions between a gaseous outer envelope, an inner icy envelope, and a rock core. As this assumption by itself restricts the possible internal density distributions, Marley et al. (1995) and Podolak et al. (2000) created random density distributions which, when reproducing the observed gravity field, could then be evaluated according to pressure-density relations of likely materials. Another possibility is to use an analytic function with sufficiently many free coefficients to adjust to the given constraints (Helled et al., 2010). The resulting pressure-density relation can be considered an empirical EOS. The advantage of the two latter methods is that they can allow for, and one can constrain the locations of, continuous (rather than sharp) density gradients. On the other hand, the resulting random or fitted density distributions can be unrealistic in a sense of not representing any composition of real materials. We here use the physical EOS LM-REOS for $\mathrm{H}, \mathrm{He}$, and $\mathrm{H}_{2} \mathrm{O}$ (Nettelmann et al., 2008) for the envelope material and the rock EOS by Hubbard and Marley (1989) for the core. This combination was also applied to the Uranus and Neptune models in Fortney and Nettelmann (2010) (hereafter FN10).

In this paper, we use the suggested modified periods and equatorial radii and the physical LM-R EOS to compute new three-layer interior and evolution models of Uranus and Neptune. In $\S 2$ we describe our method of model construction and the observational data used. In $\S 3$ we compare the resulting models obtained with the modified data to those with the Voyager data. The results are discussed and summarized in $\S 4$.

\section{Method of Structure and Evolution Modeling}

Our Uranus and Neptune models consist of three layers; a rocky core surrounded by two adiabatic and homogeneous envelopes of hydrogen, helium, and water. The interior model uses the following physical data: the planetary total mass $M_{p}$, the equatorial radius $R_{\text {eq }}$, the temperature at the 1 bar pressure level $T_{1}$ which determines the internal adiabat, the rotation rate $\omega=2 \pi / P$ with $P$ being the solid-body rotation period, and the measured gravitational coefficients $J_{2}, J_{4}$. The physical parameters used in the models are listed in Table 1. For simplicity, it is assumed that both Uranus and Neptune are adiabatic and convective, although Uranus may not have an adiabatic temperature gradient (Podolak et al., 1991). The resulting model parameters of interest here are the mass fraction of heavy elements in the inner envelope, $Z_{2}$, and in the outer envelope, $Z_{1}$, where we restrict $Z_{1}$ to $\geq 1 Z_{\odot}, Z_{\odot}=0.015$ being the solar metallicity (Lodders, 2003), the core mass, $M_{\mathrm{c}}$, and the possible transition pressure $P_{1-2}$ between the envelopes. The resulting values $Z_{1}, Z_{2}$, and $M_{\mathrm{c}}$ are obtained by an iterative scheme that fits $J_{2}, J_{4}$ and the condition of mass conservation $m=0$ at the center $r=0$. A constant He:H mass ratio of 0.275:0.725 is assumed in the envelopes. The method used to calculate interior models is the same as in (Nettelmann et al. 2008, FN10) and the thermal evolution procedure the same as in FN10. The 
progress over that work is mainly the application of improved observational data. Recent work by Jacobson and collaborators provided new estimates for the gravitational harmonics of both Uranus and Neptune using Earth-based astrometry and observations acquired with the Voyager spacecraft. Their more accurate determinations included more astrometric observations over the years, a change in reference frame to the International Celestial Reference Frame, a modification in the numerically integrated Proteus orbit (for Neptune), and an improved data processing procedure (see Jacobson (2009) for details). As in Helled et al. $(2009,2010)$ we here use the Jacobson $(2007,2009)$ data $^{1}$ for the gravitational moments $J_{2}$ and $J_{4}$. These are provided at reference equatorial radii as given in Table 1. The gravitational moments at the equatorial 1-bar radius to be fitted by our models are then obtained from the measured values (primed quantities) by scaling according to the relation $R_{\mathrm{eq}}^{2 n} J_{2 n}=R_{\mathrm{eq}}^{\prime 2 n} J_{2 n}^{\prime}$. Figure 1 shows these improved data in comparison with the former Voyager gravity data, and the relative observational uncertainties for all four outer planets.

[Table 1]

[Figure 1]

For the thermal evolution we make the standard assumption that the planet cools down over time by the release of gravitational and internal energy remnant from their formation, where the heat from the interior is transported by convection along an adiabatic temperature gradient. Thus our assumption of different heavy element mass fractions in the two envelopes, $\left(Z_{1} \neq Z_{2}\right)$ implies that one compositional gradient exists that inhibits convection across the layer boundary, but not the heat flow.

With the cooling time $\tau$ we denote the time the planet needs to cool down from an arbitrarily hot initial state to its current effective temperature $T_{\text {eff }}$. To calculate $\tau$, we pick a certain structure model (meaning a set of stationary parameters $\left\{Z_{1}, Z_{2}, m_{1-2}, M_{\mathrm{c}}\right\}$, where $\left.m_{1-2}=m\left(P_{1-2}\right)\right)$, and calculate $\sim 60$ internal profiles with stepwise increased $T_{1}$ values, that represent the planet at earlier times. We then integrate the cooling equation $L_{\mathrm{eff}}-L_{\mathrm{eq}}=-d E_{\mathrm{int}} / d t$ over time, where $L_{\text {eff }}$ is the measured infrared luminosity, $L_{\text {eq }}$ the insolation, and $d E_{\text {int }}$ the lost intrinsic energy during the time interval $d t$. To close the relations, we use the model atmosphere approximation by Guillot et al. (1995), and the Stefan-Boltzmann law to relate $T_{1}$ to $L_{\text {eff }}$. While the underlying grid for non-irradiated, solar metallicity model atmospheres by Graboske et al. (1975) may appear inappropriate for application to Uranus and Neptune despite its former application (Hubbard and MacFarlane, 1980, FN10), the induced uncertainty in the resulting cooling times of Uranus and Neptune compared to those based on a more self-consistent grid for Uranus and Neptune has been found to be $\sim 0.2$ Gyr only (Fortney

\footnotetext{
${ }^{1}$ see also the NASA/JPL website http://ssd.jpl.nasa.gov/?gravity_fields_op
}

et al., 2011). Both grids neglect the presence of clouds and hazes which are thought to be present in the tropospheres (Gautier et al., 1995). We note that clouds are just at the beginning of being incorporated into climate models for the Earth (Dessler, 2010). Therefore, the full uncertainty on the cooling time from the application of the model atmosphere grid is not known but future work on cloudy skies is not expected to significantly alter the main findings of this work as we are searching for differences between Uranus and Neptune. This also justifies the neglection of the time-dependence of the insolation, of the rotation rate due to angular momentum conservation, and of the energy of rotation. Their effects on the cooling time have been shown to nearly compensate each other in case of Jupiter (Nettelmann et al., 2012).

\section{Results}

\subsection{Models with the Voyager shape and rotation data}

Figure 2 shows our results for the envelope metallicities of three-layer Uranus and Neptune models with the Voyager shape and rotation data. There are no solutions with $Z_{1} \geq Z_{\odot}$ outside the respective boxes, as either the core mass becomes zero, or $\left|J_{4}\right|$ becomes too low or too high.

Models with the same transition pressure show a linear dependence between $Z_{1}$ and $Z_{2}$, because to first order, the resulting decrease in the outer envelope's mass $\left(d M_{1}\right)$ is proportional to the imposed reduction $-d Z_{1}$. Mass conservation then requires $d M_{2}+d M_{\mathrm{c}}=-d M_{1}$, where $d M_{2} \sim d Z_{2}$. Consequently, $d Z_{2} \sim-d Z_{1}$. If mass is shifted downward $\left(d M_{1}<0\right),\left|J_{4}\right|$ must decrease. Thus a lower limit of $Z_{1}$ at given $P_{1-2}$ arises from the condition that $\left|J_{4}\right|$ must not become too small. Since $M_{\mathrm{c}}$ decreases with increasing $Z_{2}$ in standard three-layer models as known for Jupiter (Nettelmann et al., 2012), at very high $Z_{2}$ values $(\gtrsim 0.95)$ the core mass decreases to zero before the lower observational limit of $\left|J_{4}\right|$ is reached. Thus the Neptune models along the upper right boundary in Fig. 2 are in fact two-layer models with no core. The internal adiabat then reaches temperatures up to $6600 \mathrm{~K}$ and pressures up to $8 \mathrm{Mbar}$, while the lowest core-mantle boundary temperature, $T_{\mathrm{c}}$ (pressure, $P_{\mathrm{c}}$ ) is $5200 \mathrm{~K}(5.5 \mathrm{Mbar}$ ), found for the model with the biggest rock core of mass $M_{\mathrm{c}}=3.7 M_{\oplus}$.

The described behavior is the same as found previously for Uranus and Neptune (see figure 3 in FN10). However, due to the large observational error bars of $J_{2}$ and $J_{4}$ (Fig. 1) allowed for in FN10, the Neptune models were arbitrarily forced to have $Z_{1} \leq 0.40$ and $P_{1-2} \geq 0.15 \mathrm{Mbar}$ in accordance with the results for Uranus. It is the much -reduced error bars of $J_{2}$ and $J_{4}$ that here allow us to present the full range of models. The biggest difference to the set of Neptune models in FN10 is the existence of models with pure water envelopes. Those occur for high outer envelope metallicities $Z_{1}>0.5$. Then, $P_{1-2}$ needs to be put deep inside the planet $(P \sim 1 \mathrm{Mbar})$ to ensure that $\left|J_{4}\right|$ does not get too large. 
Few Uranus models can be found for the improved error bars of $J_{2}$ and $J_{4}$ (Fig. 1). They have a well defined $Z_{2}=0.9 \pm 0.02$, a tightly constraint position of the layer boundary at $0.12 \pm 0.04$ Mbar corresponding to $r=0.79 \pm 0.02 R_{\mathrm{U}}$ and $m=0.93 \pm 0.02 M_{\mathrm{U}}$, a rather low outer envelope metallicity $1 Z_{\odot} \leq Z_{1} \leq 12 Z_{\odot}$, a rock core of at most $1 M_{\oplus}$, and central temperatures $T_{\mathrm{c}} \sim 6000 \mathrm{~K}$. An example is model U1 (Table 2). In $Z_{1}-Z_{2}$ space, the Uranus models are within the range of the Neptune models.

\section{[Figure 2, top left]}

[Figure 3, top right]

\subsection{Models with the modified shape and rotation data}

The explanations to Fig. 2 in $\S 3.1$ apply also to Fig. 3. We here describe the differences that arise from using the modified shape and rotation data.

Most obvious, the sets of solutions shift into opposite directions: the Uranus models move to the lower right corner of Fig. 3 where $Z_{2}$ gets higher and $Z_{1}$ smaller, increasing the density difference between the envelopes. In contrast, the Neptune models stretch into the upper left corner where $Z_{1}$ is above $60 \%$ and the density difference to the deep envelope less pronounced. As a result, the sets of solutions become disjunct. The Uranus solutions are tightly constrained to have a low outer envelope metallicity $(<0.1)$, a high inner envelope metallicity $(>0.9)$, a transition far out (at $\left.>0.9 M_{\mathrm{U}}\right)$, and a small core $\left(<1 M_{\oplus}\right)$. An example is model U2, see Table 2. Similar Uranus and Neptune solutions are still possible, for instance with $Z_{1} \sim 0.1$ and $Z_{2} \sim 0.88$ in both planets. However, this would require an envelope transition in Neptune at $\sim 0.05$ Mbar, relatively far out at $0.88 R_{\mathrm{N}}$ in the neutral, molecular fluid part, which is not a preferred solution for Neptune. The new finding here is that two kinds of qualitatively different Neptune models are possible that both differ in the atmospheric heavy element abundance to an observationally significant level from the Uranus models. The first kind of Neptune models are characterized by a rather large core $\left(M_{\mathrm{c}} \sim 3 M_{\oplus}\right)$ and a modest heavy element difference between the envelopes (changing from from 0.6 to 0.8 ). Alternatively, small core models are possible with pure water envelopes and a transition deep inside at $\sim 0.6 R_{\mathrm{N}}$ and $\sim 0.6 M_{\mathrm{N}}$. Models $\mathrm{N} 2 \mathrm{a}$ and $\mathrm{N} 2 \mathrm{~b}$ are respective examples (Table 2 ).

These changes can easily be explained by the new rotation rates. A slower rotation (Neptune) means a lower centrifugal force. Matter is then less strongly pushed to the outer region. If the $J_{2}, J_{4}$ to be fitted remain about the same, a higher metallicity in the outer part of the planet is required. Along the adiabat, a higher metallicity leads to lower internal temperatures. Therefore, Neptune models with high outer envelope metallicity can become rather cold, with core-mantle boundary temperatures around $5000 \mathrm{~K}$ only.

\section{[Table 2]}

Example density profiles. The input and resulting parameters of representative models are given in Table 2 and their density and mass profiles shown in Fig.4. The Uranus models clearly stand out by their big jump in density at $\sim 75 \%$ of the planet's radius, where the density falls down to $30 \%$ of the inner envelope boundary value. With the end of the inner envelope, $90 \%$ of the total mass is already accumulated. For Neptune, a model can be found (N2b) where the density difference is only $20 \%$ and thus more similar to the transition in standard three-layer Jupiter models (10\%, Nettelmann et al., 2012) than to Uranus. For Neptune model N2a, the transition occurs deep inside at $60 \%$ of the radius, where $57 \%$ of the total mass is accumulated, which would be more similar to standard three-layer models of Saturn (Nettelmann, 2009) than to any other outer planet. For both planets, a typical mean density in the inner envelope is $3 \mathrm{~g} \mathrm{~cm}^{-3}$. This (arbitrary) density threshold occurs at about $0.5 R_{p}$ (Fig. 4, left panel) and $0.4 M_{p}$ (Fig. 4, right panel), and corresponds to pressures of 2-3 Mbars and temperatures of 4000-5000 K (Fig. 5).

\section{[Figure 4] \\ [Figure 5]}

Machine-readable tabulated interior profiles of mass, pressure, radius, temperature, and density of the models $\mathrm{U} 1, \mathrm{U} 2, \mathrm{~N} 1$, and N2b are provided as supplemental onlinematerial to this article. Table 3 show an example of such a table.

\section{[Table 3]}

Moment of Inertia. The above described dichotomy in the internal structures, which mainly refers to the outer $50 \%$ of the planet's radius, maps onto different nondimensional moments of inertia ${ }^{2}, \lambda$. For Uranus and Neptune, respectively, we calculate values of $\lambda=0.2224(1)$ and $\lambda=0.2555(2)$ when using the modified shape and rotation data, while in the unmodified case the respective values are $\lambda=0.230(1)$ and $\lambda=0.2410(8)$, where the number in parenthesis gives the uncertainty in the last digit, see also Figs. 2 and 3.

\subsection{Thermal evolution}

The cooling times for the representative models are given in Table 2. The modified rotation and shapes have essentially no effect on the cooling time. This is to be expected as the cooling time mainly depends on the specific heat $c_{v}$ of the bulk material, while the composition (and hence $c_{v}$ ) is little influenced by the shape and rotation data. Interestingly, Neptune appears to be the outer

\footnotetext{
${ }^{2} \lambda=I /\left(M_{p} R_{\text {mean }}^{2}\right)$, where $I$ is the dimensional axial moment of inertia.
} 
planet with the cooling time that best matches the age of the solar system, $\tau_{\odot}=4.56 \mathrm{Gyr}$. Under the assumption of adiabatic, homogeneous cooling, Saturn's theoretical cooling time is systematically too short by 2-2.5 Gyrs (Guillot et al., 1995; Fortney and Hubbard, 2003), while Jupiter's is often found to be a little too long (Saumon et al., 1992; Guillot et al., 1995; Fortney et al., 2011). We here recover the well-known far too long cooling time of Uranus (Fortney et al., 2011), indicating that some part of the interior is not in a state of efficient energy transport through vigorous convection (Podolak et al., 1991; Hubbard et al., 1995). To estimate the size of the stable internal region in Uranus, we re-calculate the cooling time for the same quasi-adiabatic three-layer models as before but exclude a central mass, the cut-off mass $m_{\text {cut }}$, from efficient cooling. The efficiency factor $f$ quantifies the heat escape from the stable interior beneath $m_{\text {cut }}$ in comparison with the convective heat flux, so that $f=0$ implies zero heat flux and $f=1$, as well as $m_{\text {cut }}=0$, is the uninhibited case. In Fig. 6 we present the cooling time of Uranus in dependence on $m_{\text {cut }}$ and $f$.

\section{[Figure 6]}

The larger $m_{\text {cut }}$, i.e. the smaller the convective outer region, the shorter the cooling time. For a minimum cut-off mass of $0.45 M_{\mathrm{U}}, \tau=\tau_{\odot}$ can be achieved. The larger the heat flux from the stable interior, the longer the cooling time, and thus the larger the required cut-off mass. A maximum value for $f$ is obtained when $m_{\text {cut }}$ approaches $M_{\mathrm{U}}$ to realize $\tau=\tau_{\odot}$. While this maximum is $f=0.5$, we consider small values $f<0.1$ more realistic as the heat transport in a stable interior is predicted to be a tiny fraction of the convective heat flux. For Uranus, a lower limit for $f$ can be the ratio $F_{\text {cond,ad }} / F_{\text {conv }} \approx 0.02$, where $F_{\text {cond,ad }}$ is the conductive heat flux along an adiabatic gradient, and $F_{\text {conv }}$ the heat flux if Uranus would have cooled down to its present luminosity by large-scale convection (Podolak et al., 1995). Thus we conclude from figure 6 an ending of the stable interior in Uranus at $0.45-0.5 M_{\mathrm{U}}$. For comparison, we have applied the same procedure to Neptune (model N2a). Neptune's cooling time appears weakly sensitive to the innermost $20 \%$ of Neptune's mass.

\section{Discussion and Conclusions}

\subsection{Bulk composition}

We had to make several simplifications to be able to calculate Uranus and Neptune models with state-of-the art methods. One such simplification is the representation of heavy elements in the envelopes by water and the confinement of rocks to the core. In real Uranus and Neptune, silicates may also occur in the envelopes. It is clear that our assumption of having no rocks in the envelopes leads to an overestimation of the envelope metallicities and the resulting ice to rock ratio (I:R). In addition, the smaller the core mass, the larger the I:R ratio. Models with I:R $\gg 2.7$, the solar system value, may potentially invalidate this simplification and point to the presence of silicates in the envelopes. Example models are the Neptune models with pure water envelopes (N2b) and all our Uranus models. Interestingly, some Neptune models, e.g. N1 and N2a, have a rather large core $\left(\sim 3 M_{\oplus}\right)$ with a reasonable overall $\mathrm{I}: \mathrm{R}$ ratio of 1.5 times the solar value. On the other hand, our representation of ices by a pure water EOS likely overstimates the density of the true mixture of ices, and thus somewhat underestimates the mass fraction of ices that would be composed of a mixture of $\mathrm{H}_{2} \mathrm{O}, \mathrm{CH}_{4}$, and $\mathrm{NH}_{3}$. Unfortunately, high-quality EOS of light ices at pressures higher than 2 Mbar for planetary modeling are not yet available.

Under the assumptions and simplifications of this work, the bulk mass of heavy elements is $12.5 M_{\oplus}$ for Uranus and $14-14.5 M_{\oplus}$ for the selected Neptune models. This is in good agreement with the empirical EOS based models by Helled et al. (2011), who can explain the polynomial density distributions of their Uranus (Neptune) models by $\sim 11-13 M_{\oplus}\left(\sim 13-15 M_{\oplus}\right)$ of heavy elements.

\subsection{Implications from a stable deep interior}

If some part of the interior, for Uranus possibly $0.45-$ $0.5 M_{\mathrm{U}}(\S 3.3)$, is stable to convection so that heat cannot escape efficiently from the region below, then the superadiabaticity of the temperature gradient there can be nonnegligible (Leconte and Chabrier, 2012). A warmer deep interior will require a lower particle number density in order to conserve the pressure gradient. Otherwise, the induced higher warm-dense-matter pressure would cause a larger planet radius. For a given composition in the inner envelope (e.g. the $Z_{2}$ value of the adiabatic case), one might think of a lower particle number density to imply a lower envelope mean density (compared to the adiabatic case), resulting into a larger rock core mass to ensure mass conservation. However, the mean density in the inner envelope is roughly constrained by the measured $J_{2}$ value, see Fig. 4. Therefore, the metallicity in the deep interior cannot have the same $Z_{2}$ value as in the adiabatic case. Indeed, if the stable region is caused by a compositional gradient (Hubbard et al., 1995), the deep interior would have an average metallicity $Z_{3}>Z_{2}$, which may lead to a smaller rock core. Therefore, predictions on a change of the rock core mass are not possible without more sophisticated models that take into account both a compositional gradient and super-adiabaticity. At the current level of our models, the net effect of a stable interior would be the introduction of a third, deep envelope with $Z_{3}>Z_{2}$. In case of a significant super-adiabaticity in Uranus, this $Z_{3}$ might raise to $100 \%$. Moreover, a $\mathrm{H} / \mathrm{He}$-free deep interior might then be possible even with a material density larger than that of water, i.e. with an ice-rock mixture. Thus, a strongly superadiabatic deep interior (below about $\left.0.5 M_{\mathrm{U}}\right)$ may qualitatively allow for I:R ratios closer to the solar and at the same time for a $\mathrm{H} / \mathrm{He}$-free deep interior, 
resembling a traditional protoplanetary core of a few $M_{\oplus}$ that can accrete its gaseous envelope within a few million years (Hori and Ikoma, 2010).

A stable deep (i.e., far below the outer/inner envelope boundary) interior offers an explanation for the measured atmospheric helium and deuterium abundances. Given that $\mathrm{D} / \mathrm{H}$ can be enriched in the cold ices of the protosolar nebula, the observed enhanced atmospheric D:H ratio could result from upward-transport of deuterium from an ice-rich interior (Gautier et al., 1995) that is located between the inner/outer envelope boundary and the stable deep interior, where the magnetic field is believed to be generated, see $\S 4.3$. Assuming that hydrogen and helium exist in the deep interior at Mbar pressures where hydrogen is metallic, Hubbard et al. (1995) suggest that helium could phase separate and rain down leading to a helium-depleted atmosphere. However, if the particle exchange with the upper regions is suppressed in the deep interior, the rained out helium will not be replenished from the reservoir above so that the atmospheric He:H ratio remains protosolar as observed. However, we caution against taking the observed D and He abundances for clear indications of a stable deep interior, since helium phase separation from hydrogen must not necessarily occur in a metallic environment. Lorenzen et al. $(2009,2011)$ calculated the $\mathrm{H}$-He immiscibility regions in dependence on temperature, pressure, and helium concentration. In metallic hydrogen, demixing requires a sufficiently high helium concentration to be energetically preferred. For instance, at 2 Mbar our Uranus and Neptune models have a temperature of 3800$4500 \mathrm{~K}$. The phase diagram of Lorenzen et al. (2011) predicts demixing under these conditions only if the He concentration is above $\sim 1 \%$. Using

$$
\frac{N_{\mathrm{He}}}{N}=\left(1+\frac{m_{\mathrm{He}}}{m_{\mathrm{H}}} \frac{X}{Y}+3 \frac{m_{\mathrm{He}}}{m_{\mathrm{H}_{2} \mathrm{O}}} \frac{Z}{Y}\right)^{-1},
$$

where the $m_{i}$ denote the molar masses of $\mathrm{H}, \mathrm{He}$, and water, we calculate helium particle concentrations $N_{\mathrm{He}} / N$ of $1.7-3 \%$ for $Z=0.9-0.95$. This is only slightly above the demixing condition in pure H-He mixtures. As the demixing behavior of helium in more complex mixtures such as $\mathrm{H}-\mathrm{He}-\mathrm{H}_{2} \mathrm{O}$ is unknown, we cannot rule out miscibility of He the deep interior of Uranus and Neptune, be it stable (Uranus case) or unstable (Neptune case) as an explanation for the observed abundances. We encourage future work on the miscibility behavior of planetary mixtures, such as started by, e.g., Chau et al. (2011); Wilson and Militzer (2012a,b).

\subsection{Luminosity}

The modified shape and rotation data do not affect the cooling behavior. With Uranus, this is to be expected, since former calculations with various different structure models as allowed by the large error bars in $J_{2}$ and $J_{4}$ produced an uncertainty of about 1 Gyr in the evolution only, smaller than the uncertainty induced by the observational error bar of $T_{\text {eff }}$ (Fortney et al., 2011). With Neptune, this is of some surprise, since the new models are $\sim 1000 \mathrm{~K}$ colder in the deep interior than former models used for cooling curve calculations (FN10). However, the cooling curve depends on changes of temperature with time. These are similar right after the rapid cooling at young ages.

As the heat stored in the interior after formation depends on the specific heat of the bulk material, where $c_{v}$ is smaller for rocks than for ices, future work should include the admixture of rocks into the envelopes and investigate the maximum possible shortening of Uranus' cooling time within the approach of homogeneous, adiabatic evolution.

Magnetic field models of Uranus and Neptune require that $60-70 \%$ of the region interior to the ionic water layer, corresponding to $0.42-0.56 R_{p}$ (Redmer et al., 2011), is stable to convection (Stanley and Bloxham, 2006). Using our interior models, this corresponds to $0.25-0.5$ of the planet's mass. Indeed we can reproduce Uranus' measured luminosity if we assume that the heat flux from within $0.45-0.5 M_{p}$ is a negligible fraction of the heat flux in case of a convective, adiabatic interior. This consistency was already noted by Podolak et al. (1991). However, this mass or radius level is not supported by any of our Uranus structure models. Even if a density gradient occurs continuously rather than as a sharp transition, our structure models for Uranus predict a location farther out at $0.9 M_{\mathrm{U}}$. Also for Neptune, a stable interior up to $0.25-0.5 M_{p}$ is not supported by our structure models, where the deepest strong density gradient can occur at $0.57 M_{\mathrm{N}}$. Neptune's luminosity is best explained by the absence of a stable region. Future work should aim at finding consistent solutions for the structure, thermal evolution, and and magnetic field generation of Uranus and Neptune. At the current stage, the envelope separation (density gradient) of our structure models does not seem to be directly related to the luminosity, nor to the magnetic field generation.

\subsection{Why a dichotomy?}

Beside the low luminosity, Uranus differs from Neptune in having a high obliquity, dense narrow rings and its five largest satellites on regular orbits, while Neptune has a more Saturn-like obliquity and extended dusk disk with diffuse rings, and two major satellites (Triton and Nereide) on irregular orbits. These observed properties point to different formation histories and as a consequence, also to different internal structures. As discussed by Stevenson (1986), the stochastic process of impacts of various sizes and obliquities may have caused the differences we see today. A composition gradient in a heavy-element-rich giant planet could be a remnant of the formation process (Podolak et al., 1991) that has been disturbed in Neptune by a last big direct impact but not so in Uranus (an oblique impact). We are not the first authors to suggest a dichotomy between the internal structures of Uranus and Neptune. But our structure models are the first ones to confirm this pre-Voyager hypothesis of their different 
structures also on the basis of computed moment of inertia values, with an absolute difference of $d \lambda \sim 0.03(\sim 0.01)$ for the models with the (un-)modified shape and rotation data.

\subsection{A Vote for improved observational data}

New measurements of Uranus and Neptune's physical parameters could improve our knowledge of their bulk compositions and internal structures considerably. For instance, the outer envelope metallicity is rather sensitive to the assumed solid-body rotation period. While the modified periods used in this paper are perhaps not the correct ones for Uranus and Neptune, since differential rotation is possible and the winds may not be in a state of minimum energy, the presented models clearly indicate the need for more data. Recently, Karkoschka (2011) analyzed a collection of atmospheric circulation data compiled from Voyager 2 and HST observations of Neptune's atmosphere over a time-span of 20 years and found a rotation period close to the Voyager value, based on the stability of the motion of high-altitude clouds. Better determination of the rotation periods, shapes, Uranus' luminosity, Neptune's gravity field, and of the envelope metallicities below the water cloud level are indispensable for a better understanding of the interior, evolution, and formation of the icy planets in the outer solar system. We encourage the application of various discovery methods in the future, and in particular, to fly space missions to Uranus and Neptune.

\subsection{A dichotomy in the atmosphere compositions?}

One of the indications for a dichotomy in the interior structure is the possible difference in the outer envelope metallicities $Z_{1}$, which are at most $0.08(\leq 0.18)$ for Uranus but up to $0.65(\leq 0.54)$ for Neptune, using the (un-)modified shape and rotation data. In case of vertical mixing up into the troposphere, one would expect to see a signature from different outer envelope metallicities in the lower atmosphere. Over the past 60 years, several attempts of deriving the atmosphere abundances from measured spectra have been undertaken, see, e.g., the reviews of Fegley et al. (1991); Gautier et al. (1995); Guillot and Gautier (2007). However, that turned out to be quite challenging, mostly because of the numerous absorption lines of $\mathrm{CH}_{4}$ and $\mathrm{H}_{2}$, uncertainties in the line shapes and absorption coefficients, non-equilibrium chemistry, and the dependences of the derived abundances among each other. Today, carbon is the heavy element for which the interpretation of the available data gives the least ambiguous picture. A value of $\mathrm{C}: \mathrm{H}=30-60 \times$ solar for both planets has finally emerged (Gautier et al., 1995). Assuming $\mathrm{C}: \mathrm{H}=30 \times$ solar and similar enrichments also of $\mathrm{N}$ and $\mathrm{O}$, Hubbard et al. (1995) estimate an outer envelope ice mass fraction of 0.08 . While this is in good agreement with the possible $Z_{1}$ values of our Uranus and Neptune models, it does not support our high- $Z_{1}$ Neptune models as required for qualitatively different interiors. On the other hand, both $\mathrm{CO}$ and $\mathrm{HCN}$ have been detected in the atmosphere of Neptune but not of Uranus (Gautier et al., 1995), indicating different processes at work in their atmospheres. To replace assumptions by real data, we encourage deep entry probe missions to Uranus and Neptune.

\subsection{Past and present structure models}

As shown in figures 1-3, more definite conclusions about the dissimilarity of the internal structures are prevented by the current observational uncertainty in the gravitational moments. This is at odds with the struggles of earlier modelers (Podolak et al., 1995; Marley et al., 1995) to find Uranus and Neptune models at all that matched the less accurately known gravity data in the past. Moreover, despite the tighter present constraints, our models seem to encompass many of the previously found models.

In particular, Hubbard and Marley (1989) developed Uranus models with physical EOS, smooth transitions between an outer H-He rich envelope, an inner ice-rich envelope, and a rock core, and showed that models with 5-15\% $\mathrm{H}-\mathrm{He}$ in the inner envelope could give best agreement with the imposed constraints. Such an H-He abundance is also seen in our Uranus models.

When the Voyager gravity and rotation rate data for Neptune became available, Podolak et al. (1995) applied the three-layer approach and physical EOS to compute Uranus and Neptune models. Their Uranus models required some $\mathrm{H}-\mathrm{He}$ to be present in the deep interior, too, whereas for Neptune this was found to be optional (nonconventional models). They could also find a different Neptune model with an inner envelope of pure water and transition deeper inside at $\sim 0.7 R_{\mathrm{N}}$, and a highly enriched outer envelope, indicating a possible difference in the interior structures of Uranus and Neptune. Our Neptune models in the upper right corners of Figs. 2,3 are similar to that latter one, while their non-conventional models are within the bulk of our Uranus and Neptune models.

Marley et al. (1995) again allowed for smooth density gradients and generated models with random density distributions. Nevertheless, they found that a rather sharp transition from a low-density outer envelope to a highdensity inner envelope is necessary to fit the Voyager values of $J_{2}$ and $J_{4}$, where the pressure-density relation in the inner envelope could be that of an ice layer. Because of the admittance of smooth density gradients, they found for the first time that the transition could occur as deep as between $\sim 60-65 \%$ of the planet's radius. Our model N2b shows the same properties, although within the three-layer approach.

Finally, Helled et al. (2011) were the first to use the accurate post-Voyager gravity data of Jacobson (2007, 2009). Their polynomial density-profiles could well represent a metallicity gradient that rises from about solar metallicity at the surface to about $85 \%$ in the center, with slightly higher values preferred for Neptune but no indication of different internal structures. The found metallicities are within those of our models. 
According to the present work, the application of the modified shape and rotation data gives a more pronounced indication of different structure than seen so far.

\subsection{Conclusions}

We present the full sets of three-layer interior models (with $\mathrm{H} / \mathrm{He}$ /water envelopes and rocks confined to the core) of Uranus and Neptune for different solid-body rotation periods and flattenings, using the improved gravity data by Jacobson (2007, 2009), and physical equations of state. We find that the resulting bulk composition is insensitive to the current level of uncertainty in the input data (observational constraints and equations of state) as our results are in good agreement with previous calculations (e.g. Podolak et al. 1995; Marley et al. 1995; Helled et al. 2011). However, our models with the modified rotation periods and shapes suggest that Uranus and Neptune could be quite different. Uranus would have an outer envelope with a few times the solar metallicity which transitions to a heavily enriched ( $\sim 90 \%$ by mass heavy elements) inner envelope at $0.9 M_{\mathrm{U}}$, giving a rather low moment of inertia of $\sim 0.222$. In Neptune, this transition can occur deeper inside at $0.6 M_{\mathrm{N}}$ and be accompanied by a more moderate increase in metallicity, leading to a less centrally condensed planet with $\lambda \sim 0.255$. While the observed magnetic fields of Uranus and Neptune are similar and can be reproduced by a rather narrow range of dynamo models, dissimilar interiors are required to explain the measured luminosities. We have presented a new indication for different internal structures based on the application of modified shape and rotation data. However, the density gradient in our models appears to be generally farther out than required by evolution and magnetic field models.

The authors thank the two referees for providing fruitful comments that led us to consider the results in a wider context. NN acknowledges support from the DFG RE 881/111 .

\section{References}

Anderson, J., Schubert, G., 2007. Saturn's Gravitational Field, Internal Rotation, and Interior Structure. Science 317, 1384-1387.

de Bergh, C., Lutz, B.L., Owen, T., Brault, J., Chauville, J., 1986. Monoteuterated methane in the outer solar system. ii. its detection on uranus at $1.6 \mu \mathrm{m}$. ApJ 311, 501-510.

de Bergh, C., Lutz, B.L., Owen, T., Maillard, J.P., 1990. Monoteuterated methane in the outer solar system. iv. its detection and abundance on neptune. ApJ 355, 661-666.

Borucki, W.J., Koch, D., Basri, G., Batalha, N., Brown, T., Bryson, S.T., Caldwell, D., Christensen-Dalsgaard, J., Cochran, W.D., et al., 2011. Characteristics of Planetary Candidates Observed by Kepler. II. Analysis of the First Four Months of Data. ApJ 736, A19.

Brozovic, M., Jacobson, R.A., 2009. The Orbits of the Outer Uranian Satellites. Astronom. J. 137, 3834.

Chau, R., Hamel, S., Nellis, W.J., 2011. Chemical processes in the deep interior of Uranus. Nature Communications 2, A203.

Dessler, A.E., 2010. A determination of the cloud feedback from climate variations of the last decade. Science 330, 1523.
Fegley, B., Gautier, D., Owen, T., Prinn, R.G., 1991. Spectroscopy and chemistry of the atmosphere of uranus, in: Bergstrahl, J.T., Miner, E.D., Matthews, M.S. (Eds.), Uranus. University of Arizona, Tucson, p. 147.

Fortney, J.J., Hubbard, W.B., 2003. Phase Separation in Giant Planets: Inhomogeneous evolution of Saturn. Icarus 164, 228.

Fortney, J.J., Ikoma, M., Nettelmann, N., Guillot, T., Marley, M.S., 2011. Self-consistent Model Atmospheres and the Cooling of the Solar System Giant Planets. ApJ 729, 32.

Fortney, J.J., Nettelmann, N., 2010. The Interior Structure, Composition, and Evolution of Giant Planets. Space Sci. Rev. 152, $423-447$.

Gautier, D., Conrath, B.J., Owen, T., "De Pater", I., Atreya, S.K., 1995. The troposphere of neptune, in: Cruishank (Ed.), Neptune and Triton. University of Arizona, Tucson, pp. 547-611.

Graboske, H.C., Plness, R.J., Pollack, J.B., Grossman, A.S., 1975. The structure and evolution of jupiter: the fluid contraction stage. ApJ 199, 265.

Guillot, T., Chabrier, G., Gautier, D., Morel, P., 1995. Effect of radiative transport on the evolution of Jupiter and Saturn. ApJ $450,463$.

Guillot, T., Gautier, D., 2007. The giant planets, in: Schubert, G., Spohn, T. (Eds.), Treatise of Geophysics, vol. 10, Planets and Moons. Amsterdam: Elsevier, p. 439 (arXiv:0912:2019).

Helled, R., Anderson, J., Podolak, M., Schubert, G., 2011. Interior models of Uranus and Neptune. Astrophys. J. 726, A15.

Helled, R., Anderson, J.D., Schubert, G., 2010. Uranus and Neptune: shape and rotation. Icarus 210, 446.

Helled, R., Schubert, G., Anderson, J.D., 2009. Jupiter and Saturn Rotation Periods. Planet. Space Sci. 57, 1467-1473.

Hori, Y., Ikoma, M., 2010. Critical core masses for gas giant formation with grain-free envelopes. ApJ 714, 1343.

Hubbard, W.B., MacFarlane, J.J., 1980. Structure and Evolution of Uranus and Neptune. J. Geophys. Res. 88, 225.

Hubbard, W.B., Marley, M.S., 1989. Optimized Jupiter, Saturn, and Uranus interior models. Icarus 78, 102.

Hubbard, W.B., Nellis, W.J., Mitchell, A.C., Holmes, N.C., McCandless, P.C., Limaye, S.S., 1991. Interior structure of neptune comparison with uranus. Science 253, 648-651.

Hubbard, W.B., Podolak, M., Stevenson, D.J., 1995. The Interior of Neptune, in: Cruishank (Ed.), Neptune and Triton. University of Arizona, Tucson, p. 109.

Jacobson, R.A., 2003. JUP230 orbit solution.

Jacobson, R.A., 2007. The Gravity Field of the Uranian System and the Orbits of the Uranian Satellites and Rings. BAAS 39, 453.

Jacobson, R.A., 2009. The Orbits of the Neptunian Satellites and the Orientation of the Pole of Neptune. Astronomical J. 137, 4322.

Kane, S.R., 2011. Detecting the Signatures of Uranus and Neptune. Icarus 214, 327-333.

Karkoschka, E., 2011. Neptunes rotational period suggested by the extraordinary stability of two features. Icarus 215, 439-448.

Leconte, J., Chabrier, G., 2012. A new vision on giant planet interiors: the impact of double diffusion convection. Astron. Astroph. 540, A20.

Lindal, G., 1992. The atmosphere of Neptune: an analysis of radio occultation data acquired with Voyager 2. Astronom. J. 103, 967.

Lindal, G.F., Lyons, J., Sweetnam, D., Eshleman, V., Hinson, D., Tyler, G., 1987. The Atmosphere of Uranus: Results of Radio Occultation Measurements With Voyager 2. J. Geophys. Res. 92, 14,987-15,001.

Lodders, K., 2003. Solar System Abundances and Condensation Temperatures of the Elements. ApJ 591, 1220.

Lorenzen, W., Holst, B., Redmer, R., 2009. Demixing of hydrogen and helium at megabar pressures. Phys. Rev. Lett. 102, 5701.

Lorenzen, W., Holst, B., Redmer, R., 2011. Metallization in Hydrogen-Helium mixtures. PRB 84, 235109.

Marley, M., Gomez, P., Podolak, M., 1995. Monte Carlo interior models for Uranus ad Neptune. J. Geophys. Res. 100, 349-353.

Nettelmann, N., 2009. Matter under extreme conditions: modelling giant planets. Ph.D. thesis. Universität Rostock.

Nettelmann, N., Becker, A., Holst, B., Redmer, R., 2012. Jupiter 
models with improved hydrogen EOS (H-REOS.2). ApJ 749.

Nettelmann, N., Holst, B., Kietzmann, A., French, M., Redmer, R., Blaschke, D., 2008. Ab initio equation of state data for hydrogen, helium, and water and the internal structure of Jupiter. ApJ 683, 1217.

Podolak, M., Hubbard, W., Stevenson, D.J., 1991. Models of Uranus' Interior and Magnetic Field, in: Bergstrahl, J., Miner, E., Matthews, M. (Eds.), Uranus. University of Arizona Press, Tucson, p. 29

Podolak, M., Podolak, J.I., Marley, M.S., 2000. Further Investigation of random models of Uranus and Neptune. Planet. Space Sci. 48, 143.

Podolak, M., Weizman, A., Marley, M.S., 1995. Comparative models of Uranus and Neptune. Planet. Space Sci. 43, 1517-1522.

Redmer, R., Mattsson, T.R., Nettelmann, N., French, M., 2011. The phase diagram of water and the magnetic field of Uranus and Neptune. Icarus 211, 798 .

Saumon, D., Hubbard, W.B., Chabrier, G., van Horn, H.M., 1992. The role of the molecular-metallic transition of hydrogen in the evolution of jupiter, saturn, and brown dwarfs. ApJ 391, 827-831.

Stanley, S., Bloxham, J., 2006. Numerical dynamo models of Uranus' and Neptune's magnetic fields. Icarus $184,556-572$.

Stevenson, D., 1986. The Uranus-Neptune dichotomy: the role of giant impacts. preprint 1986LPI...17.1011S .

Wilson, H.F., Militzer, B., 2012a. Rock core solubility in jupiter and giant exoplanets. Phys. Rev. Lett. 108, 111101.

Wilson, H.F., Militzer, B., 2012b. Solubility of water ice in metallic hydrogen: Consequences for core erosion in gas giant planets. ApJ 745,54 . 

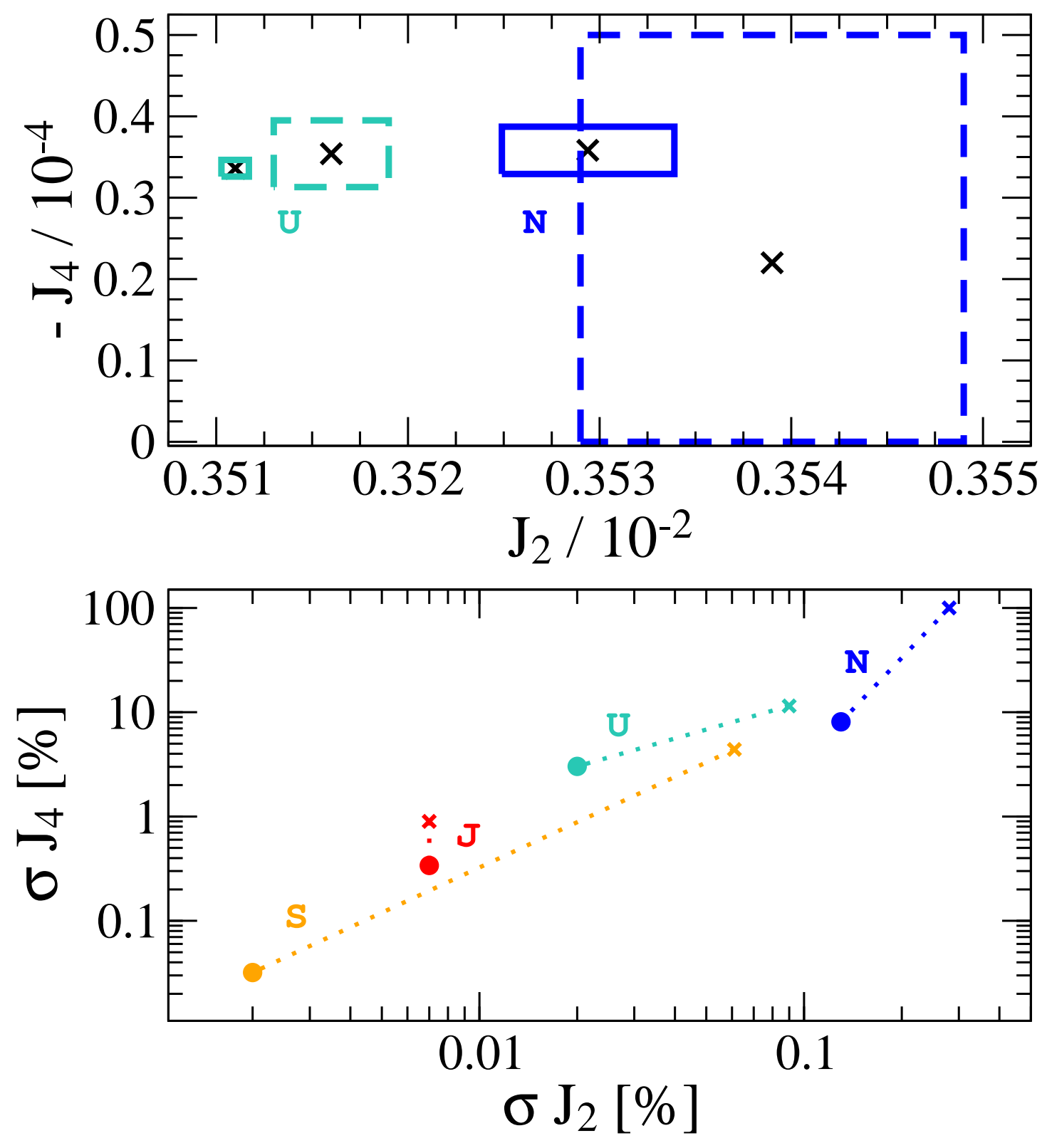

Figure 1: (Color online)

Upper panel: Observed gravitational moments $J_{2}$ and $J_{4}$ of Uranus (U, cyan) and Neptune $(\mathrm{N}$, blue) with $\mathbf{1} \sigma$ error bars according to the Voyager and pre-Voyager data (dashed) and the Jacobson data (solid). Crosses indicate the mean values. Lower panel: relative observational $1 \sigma$ uncertainties in $J_{2}$ and $J_{4}$ from the Voyager and Pioneer missions (crosses) and improved values (circles); J, red: Jupiter, improved Galileo data from Jacobson (2003); S, orange: Saturn, improved Cassini data from Anderson and Schubert (2007); U, cyan: Uranus; N, blue: Neptune. 


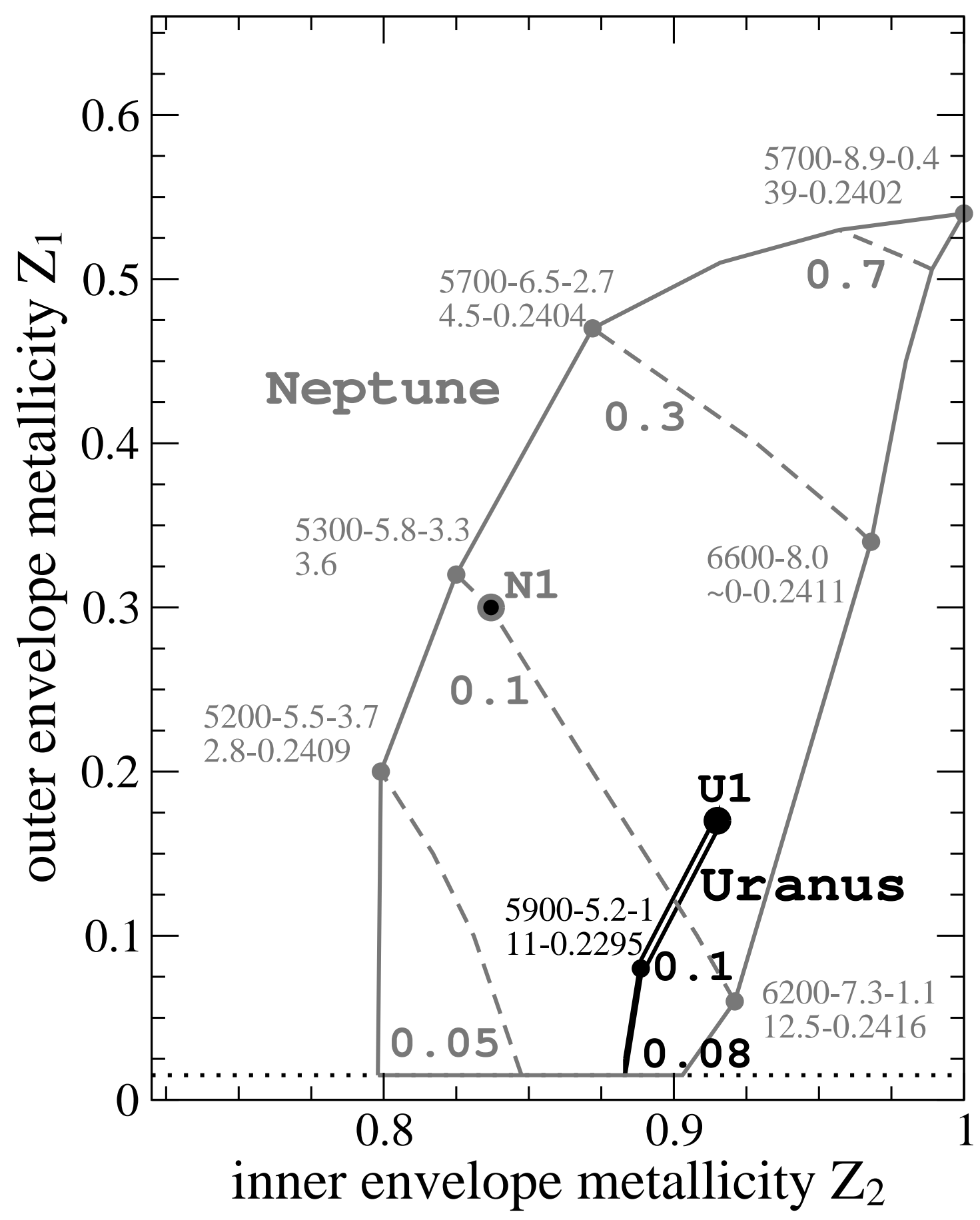

Figure 2: (Color online)

Heavy element mass fraction in the outer envelope $\left(Z_{1}\right)$ and inner envelope $\left(Z_{2}\right)$ of Uranus models (black) and Neptune models (grey) as labeled with the Voyager shape and rotation data. The solid lines frame the full set of solutions for each planet. Dashed lines within the box of Neptune models indicate solutions of same transition pressure in [Mbar] as labeled. Numbers at selected models (filled circles) give $T_{\mathrm{c}}[\mathrm{K}], P_{\mathrm{c}}[\mathrm{Mbar}], M_{\mathrm{c}}\left[M_{\oplus}\right]$, the ice-to-tock ratio I:R, and $\lambda$. The dotted line is a guide to the eye for the solar metallicity $Z_{\odot}=0.015$. 


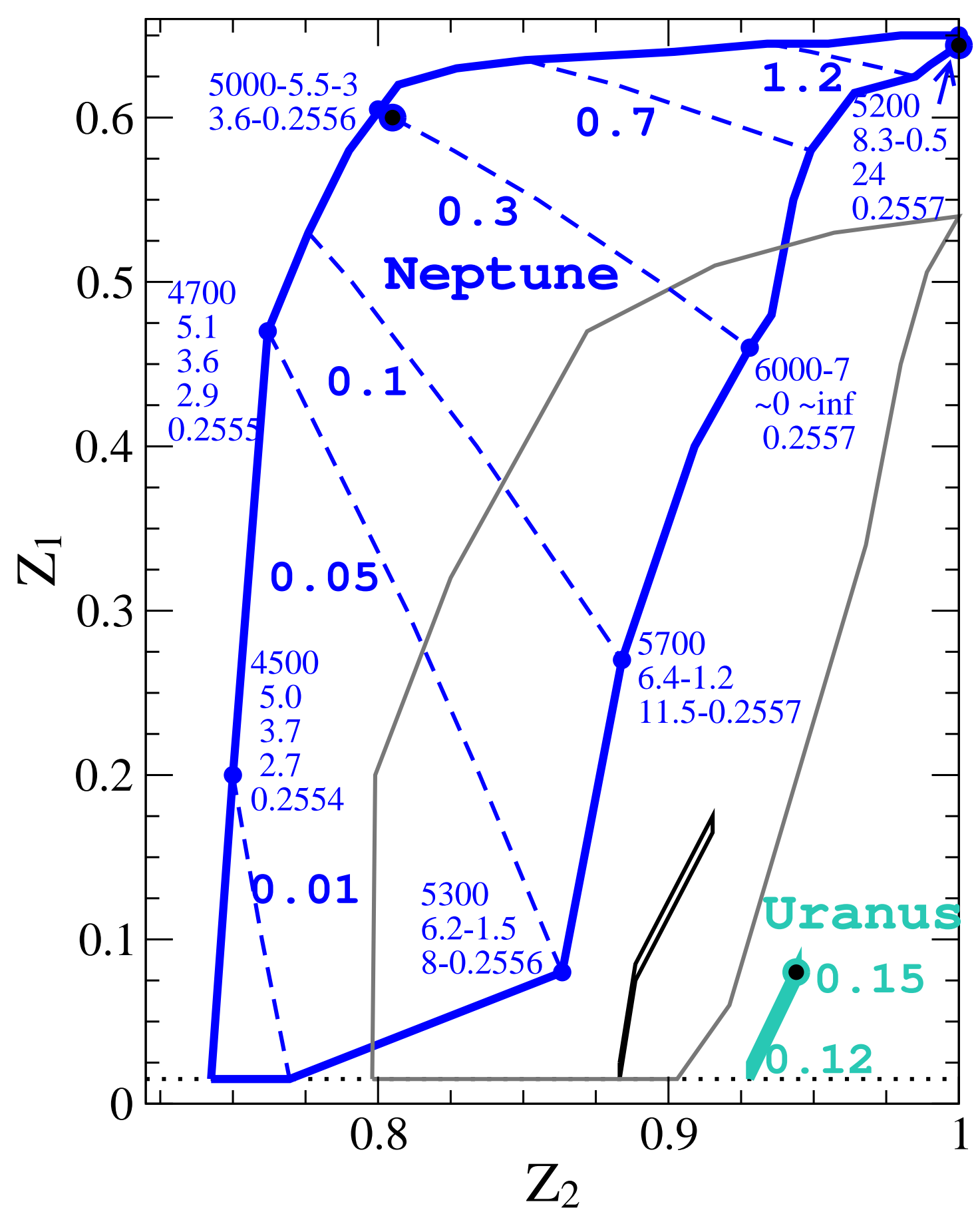

Figure 3: (Color online)

Same as Fig. 2 but using the modified shape and rotation data for Uranus (cyan) and Neptune (blue). Models U2, N2a, and N2b (Table 2) are highlighted by black-filled, big circles. The boxes of Fig. 2 are also shown to facilitate the comparison. 

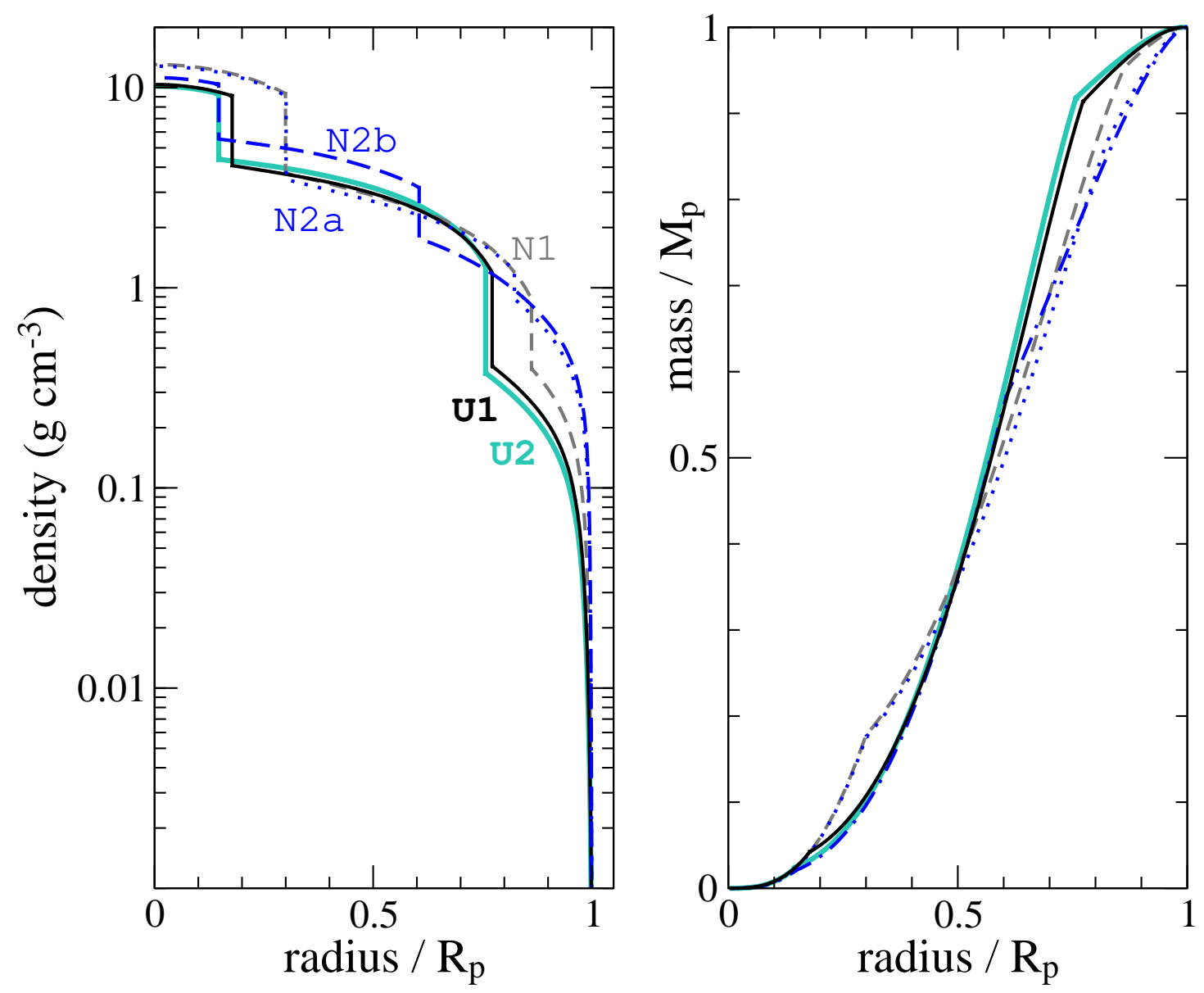

Figure 4: (Color online)

Internal density (left panel) and mass (right panel) profiles over the normalized planetary radius of the models U1 (solid, black), U2 (thick solid, cyan), N1 (short-dashed, grey), N2a (dotted, blue), and N2b (long-dashed, blue). See Table 2 for details. 


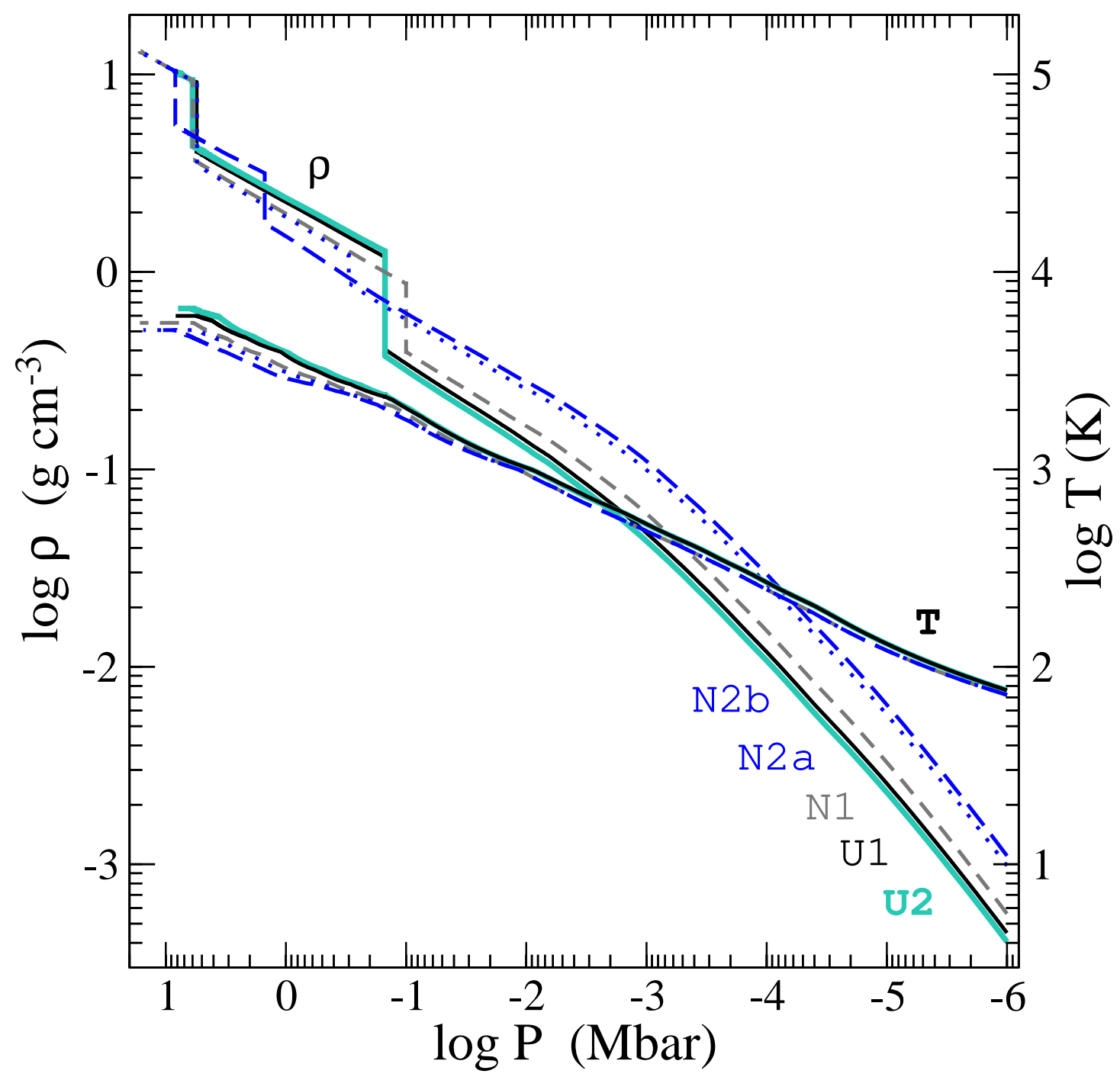

Figure 5: (Color online)

Internal density and temperature profiles over pressure. See Fig. 4 for description of line-styles and labels. 


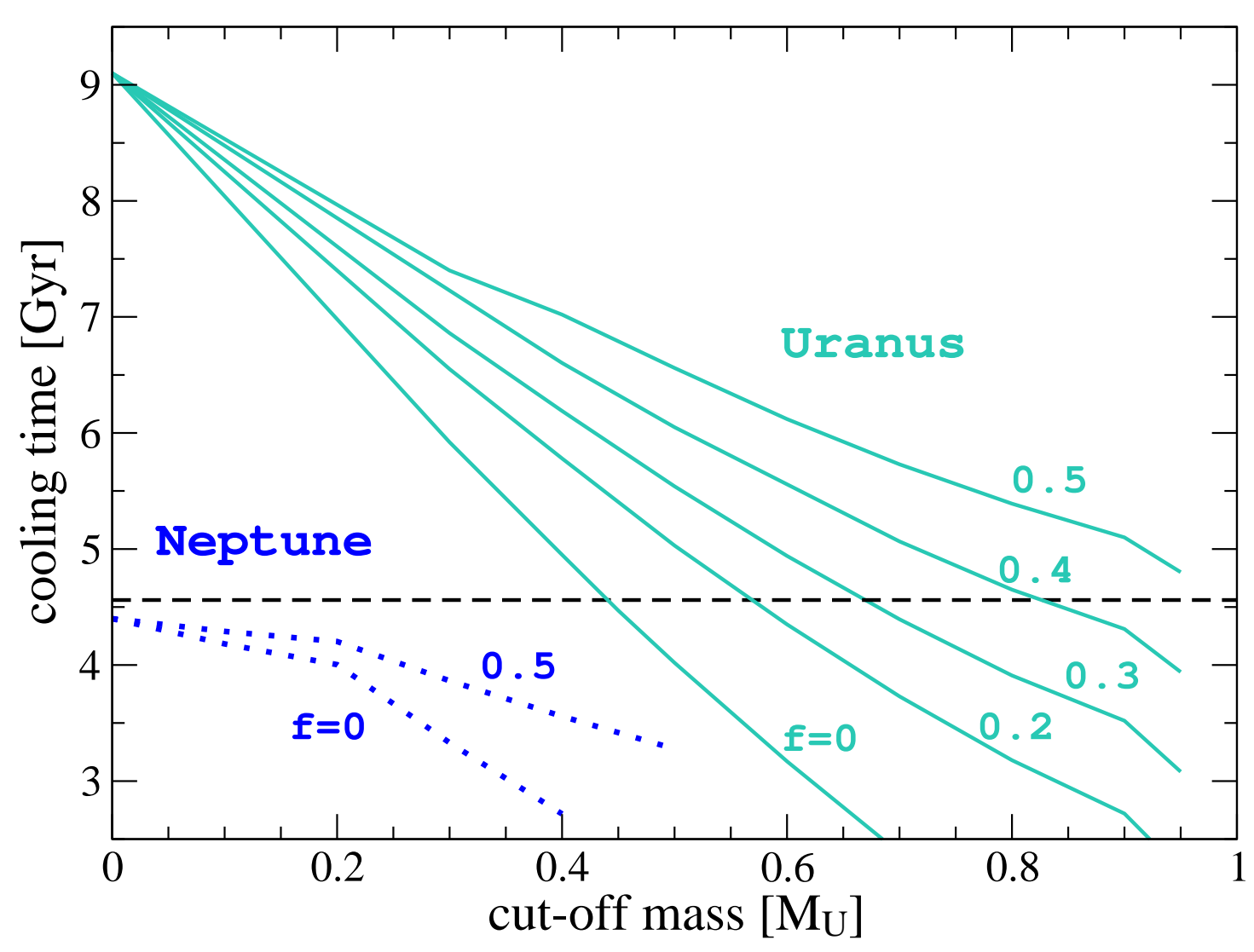

Figure 6: (Color online)

Thermal evolution of Uranus (model U2, solid, cyan) assuming that the heat flux from the mass interior to the cut-off mass ( $x$-axis) is limited to a fraction $f$ of the convective energy flux. For $f=0-0.4$ and a cut-off mass of $0.45-0.85$, a cooling time in agreement with the age of the solar system (horizontal dashed line) can be found. For comparison, we also show the same calculations for Neptune (model N2a, dotted, blue). 
Table 1: Physical constraints of Uranus and Neptune.

\begin{tabular}{|c|c|c|c|c|}
\hline Parameter & $\overline{\mathrm{U}} \mathrm{U}$ data 1 & $\overline{\mathrm{U}}$ data 2 & $\overline{\mathrm{N} \text { data } 1}$ & $\overline{\mathrm{N}}$ data 2 \\
\hline$M_{p}\left(M_{\oplus}\right)$ & 14.536 & 14.536 & 17.148 & 17.148 \\
\hline$\omega / 2 \pi$ & $17 \mathrm{~h} 14 \mathrm{~m} 40 \mathrm{~s}^{\mathrm{a}}$ & $16 \mathrm{~h} 34 \mathrm{~m} 24 \mathrm{~s}^{\mathrm{c}}$ & $16 \mathrm{~h} 6 \mathrm{~m} 40 \mathrm{~s}^{\mathrm{a}}$ & $17 \mathrm{~h} 27 \mathrm{~m} 29 \mathrm{~s}^{\mathrm{c}}$ \\
\hline$T_{1}(\mathrm{~K})$ & $76(2)^{\mathrm{b}}$ & $76(2)$ & $72(2)^{\mathrm{a}}$ & $72(2)$ \\
\hline$T_{\text {eff }}(\mathrm{K})$ & $59.1(3)^{\mathrm{g}}$ & $59.1(3)$ & $59.3(8)^{\mathrm{g}}$ & $59.3(8)$ \\
\hline$R_{\mathrm{eq}}^{\prime}$ & $26,200^{\mathrm{d}, \mathrm{e}}$ & $26,200^{\mathrm{d}, \mathrm{e}}$ & $25,225^{\mathrm{d}, \mathrm{f}}$ & $25,225^{\mathrm{d}, \mathrm{f}}$ \\
\hline$J_{2}^{\prime} / 10^{-2}$ & $0.334129(72)^{\mathrm{d}, \mathrm{e}}$ & $0.334129(72)^{\mathrm{d}, \mathrm{e}}$ & $0.340843(450)^{\mathrm{d}, \mathrm{f}}$ & $0.340843(450)^{\mathrm{d}, \mathrm{f}}$ \\
\hline$J_{4}^{\prime} / 10^{-4}$ & $-0.3044(102)^{\mathrm{d}}$ & $-0.3044(102)$ & $-0.334(29)^{\mathrm{d}, \mathrm{f}}$ & $-0.334(29)^{\mathrm{d}, \mathrm{f}}$ \\
\hline$R_{\text {eq }}(\mathrm{km})$ & $25,559(4)^{\mathrm{a}}$ & $25,559(4)^{\mathrm{c}}$ & $24,766(15)^{\mathrm{a}}$ & $24,787(4)^{\mathrm{c}}$ \\
\hline $\mathrm{J}_{2} / 10^{-2}$ & $0.351099(72)^{\mathrm{c}}$ & $0.351099(72)^{\mathrm{c}}$ & $0.35294(45)^{\mathrm{c}}$ & $0.35294(45)^{\mathrm{c}}$ \\
\hline $\mathrm{J}_{4} / 10^{-4}$ & $-0.3361(100)^{c}$ & $-0.3361(100)^{c}$ & $-0.358(29)^{\mathrm{c}}$ & $-0.358(29)^{\mathrm{c}}$ \\
\hline
\end{tabular}

Numbers in parenthesis are the observational error bars in the last digits. The gravitational moments $J_{2 n}^{\prime}$ are the measured ones. They refer to a reference equatorial radius $R_{\text {eq }}^{\prime}$. The gravitational moments $J_{2 n}$ refer to the equatorial radius at the 1-bar pressure level, $R_{\text {eq }}$.

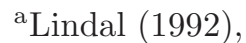

${ }^{\mathrm{b}}$ Lindal et al. (1987),

${ }^{\mathrm{c}}$ Helled et al. (2010),

dhttp://ssd.jpl.nasa.gov,

eBrozovic and Jacobson (2009),

${ }^{\mathrm{f}}$ Jacobson (2009)

bafter Guillot and Gautier (2007) 
Table 2: Five resulting structure models.

\begin{tabular}{lccccc}
\hline \hline Parameter & U1 & U2 & N1 & N2a & N2b \\
\hline$R_{\text {eq }}(\mathrm{km})$ & 25,559 & 25,559 & 24,773 & 24,786 & 24,786 \\
$R_{\text {mean }}(\mathrm{km})$ & 25,388 & 25,378 & 24,622 & 24,650 & 24,650 \\
$f$ & 0.0198 & 0.0210 & 0.0180 & 0.0163 & 0.0163 \\
$J_{2} / 10^{-2}$ & 0.35107 & 0.35107 & 0.3533 & 0.3531 & 0.3530 \\
$J_{4} / 10^{-4}$ & -0.345 & -0.344 & -0.378 & -0.383 & 0.3835 \\
$P_{1-2}(\mathrm{Mbar})$ & 0.15 & 0.15 & 0.10 & 0.30 & 1.50 \\
$m_{1-2}\left(M_{p}\right)$ & 0.913 & 0.918 & 0.953 & 0.864 & 0.571 \\
$r_{1-2}\left(R_{p}\right)$ & 0.772 & 0.757 & 0.927 & 0.823 & 0.605 \\
$Z_{1}$ & 0.17 & 0.08 & 0.30 & 0.60 & 0.644 \\
$Z_{2}$ & 0.915 & 0.944 & 0.833 & 0.805 & 1 \\
$M_{\mathrm{c}}\left(M_{\oplus}\right)$ & 0.61 & 0.36 & 3.15 & 3.02 & 0.35 \\
$T_{\mathrm{c}}(\mathrm{K})$ & 6000 & 6500 & 5500 & 5000 & 5200 \\
$P_{\mathrm{c}}(\mathrm{Mbar})$ & 5.5 & 6.0 & 6.0 & 5.5 & 8.3 \\
$P_{0}(\mathrm{Mbar})$ & 8.3 & 7.9 & 16.4 & 15.3 & 10.5 \\
$M_{Z}\left(M_{\oplus}\right)$ & 12.4 & 12.7 & 14.4 & 13.9 & 14.4 \\
$\mathrm{I}: \mathrm{R}$ & 19.2 & 35.3 & 3.7 & 3.6 & 13.7 \\
$\tau(\mathrm{Gyr})$ & 10.0 & 9.1 & 4.8 & 4.4 & 4.3 \\
$\lambda$ & 0.2296 & 0.2224 & 0.2405 & 0.2555 & 0.2557 \\
\hline
\end{tabular}

Uranus model U1 and Neptune model N1 are based on the input data sets No. 1, while models U2, N2a, and N2b are on data sets No. 2, see Table 1 . The pressure $P_{0}$ refers to $m=0$, whereas $P_{\mathrm{c}}$ to $m=M_{\mathrm{c}}$. 
Table 3: Example of a tabulated interior profile.

\begin{tabular}{ccccc}
\hline \hline $\begin{array}{c}\text { Mass } \\
\left(M_{\oplus}\right)\end{array}$ & $\begin{array}{c}\text { Pressure } \\
(\mathrm{GPa})\end{array}$ & $\begin{array}{c}\text { Radius } \\
\left(R_{\oplus}\right)\end{array}$ & $\begin{array}{c}\text { Temperature } \\
(\mathrm{K})\end{array}$ & $\begin{array}{c}\text { Density } \\
(\mathrm{g} / \mathrm{ccm})\end{array}$ \\
\hline 14.5322753 & $1.0000 \mathrm{E}-04$ & 3.979310 & 76.0 & $4.4876 \mathrm{E}-04$ \\
$\vdots$ & $\vdots$ & $\vdots$ & $\vdots$ & $\vdots$ \\
13.2697748 & $1.4999 \mathrm{E}+01$ & 3.073483 & 2338.8 & $4.0541 \mathrm{E}-01$ \\
13.2697239 & $1.5000 \mathrm{E}+01$ & 3.073465 & 2338.8 & $1.1879 \mathrm{E}+00$ \\
$\vdots$ & $\vdots$ & $\vdots$ & $\vdots$ & $\vdots$ \\
0.5987030 & $5.5226 \mathrm{E}+02$ & 0.700230 & 6083.5 & $4.0700 \mathrm{E}+00$ \\
0.5984897 & $5.5234 \mathrm{E}+02$ & 0.700034 & 6083.6 & $9.0790 \mathrm{E}+00$ \\
$\vdots$ & $\vdots$ & $\vdots$ & $\vdots$ & $\vdots$ \\
0.0000019 & $8.2114 \mathrm{E}+02$ & 0.010000 & 6083.6 & $1.0326 \mathrm{E}+01$ \\
\hline
\end{tabular}

Tables of the interior profiles of the models $\mathrm{U} 1, \mathrm{U} 2, \mathrm{~N} 1$, and N2b are published in their entirety as supplemental material in the electronic edition. A portion of the table of model U1 is shown here for guidance of the tables' form and content. The selected rows show, from top to bottom, the outer boundary, the outer/inner envelope transition, the core-mantle boundary, and the center. 